\title{
Incidence and outcome of delirium during helmet CPAP treatment in COVID-19 patients
}

\author{
Federica Samartin ${ }^{1}$ (1) $\cdot$ Emanuele Salvi ${ }^{1} \cdot$ Anna Maria Brambilla $^{1} \cdot$ Alessandro Torre $^{1} \cdot$ Stella Ingrassia ${ }^{1}$. \\ Antonio Gidaro ${ }^{1}$
}

Received: 25 April 2021 / Accepted: 18 July 2021 / Published online: 14 August 2021

(c) Società Italiana di Medicina Interna (SIMI) 2021

\section{Dear Editor,}

Coronavirus disease 2019 (COVID-19) is an infectious disease caused by severe acute respiratory syndrome coronavirus 2 (SARS-CoV-2) which may causes Acute Hypoxemic Respiratory Failure (AHRF) or Acute Respiratory Distress Syndrome (ARDS) requiring Non-Invasive Ventilation (NIV) and in particular Helmet Continuous Positive Airway Pressure (CPAP), preferred for use both as a ventilator and as a protection against viral transmission for healthcare workers.

Beyond the classical signs and symptoms of presentation as fever and cough, it is estimated that more than one-third of patients with COVID-19 develop neurologic or neuropsychiatric symptoms that seem to be associated with more severe disease [1]. Although neurologic manifestations can happen in a broad spectrum of acute infectious diseases, there are evidences suggesting that the coronavirus family is specially neurotropic, indeed many neurological complications and signs suggestive of delirium are common in the acute stage of SARS (Severe Acute Respiratory Syndrome), MERS (Middle Est Respiratory Syndrome) and even COVID-19 [2]. Delirium should be recognized as a potential feature of COVID-19 and may be the only presenting symptom, especially in dementia patients [3].

In COVID-19 patients delirium has a prognostic significance, being independently associated with higher mortality rate, increased length of stay and a greater rate of admission in Intensive Care Unit (ICU) and ventilator utilisation [3-5].

Although no data are available about a direct correlation between NIV and delirium, several well-known precipitating

Federica Samartin

federica.samartin@unimi.it

1 Department of Biomedical and Clinical Sciences Luigi Sacco, Luigi Sacco Hospital, University of Milan, Milan, Italy factors of delirium can co-exist during NIV, especially with helmet interface, as isolation, noise, contact limitation, dehydration. So much that it might be possible that prolonged CPAP could increase delirium rate.

The first aim of our study is to investigate the incidence of delirium occurring during Helmet CPAP therapy in COVID19 patients. Moreover, we wanted to verify if there are predictable risk factors for delirium in CPAP ventilated patients and to determine if delirium increases the risk of adverse outcomes, defined as need of endotracheal intubation (ETI) and death. Secondly, we better characterize delirium's risk factors including them in a Cox proportional hazard multivariate model.

We analysed data from patients admitted to the internal medicine and infectious diseases wards of "Luigi Sacco" University Hospital of Milan from 21 February to 5 May 2020 . The study is part of a prospectively conducted registry study ("REGISTRO DELLE INFEZIONI SOSPETTE E ACCERTATE COVID-19/Studio Sacco COVID-19") which was approved by the Local Ethical Committee with the Registration Number 2020/16088.

The inclusion criteria were:

1. COVID-19 pneumoniae defined by positivity of realtime reverse transcription-polymerase chain reaction test (RT-PCR) at nose-pharyngeal swab for SARS-CoV-2 and a Chest X-Ray positive for interstitial pneumonia.

2. AHRF requiring Helmet CPAP treatment with $\mathrm{PaO} 2 /$ $\mathrm{FiO} 2(\mathrm{P} / \mathrm{F}) \leq 200 \mathrm{mmHg}$ and respiratory rate $>25 / \mathrm{min}$ ute.

DSM-5 criteria were used for the diagnosis of delirium:

1. Presence of disorder of consciousness with reduced ability to focus, sustain, or shift attention.

2. Change in cognition that is not better accounted for by a pre-existing, established, or evolving dementia. 
3. Development of the disorder over a short period with fluctuation during the day.

4. Evidence that the disorder is caused by a direct physiologic consequence of a medical condition.

Kolmogorov-Smirnov test was used to evaluate the normality of data distribution. Qualitative data were expressed as number and percentage. Chi square or Fisher exact tests were used for group's comparison. Quantitative data were expressed as mean, standard deviation, median and range. Student $T$ test and Mann-Whitney test (for non-parametric data) were used for comparison between groups. $P$ value less than 0.05 was considered statistically significant. Cox proportional hazard model was used to evaluate four delirium risk factors: age, previous diagnosis of dementia or psychiatric condition, P/F soon after starting CPAP and Gr/Ly. Excel (Office program 2010) and SPSS (statistical package for social science-SPSS, Inc., Chicago, IL version 26) were used for statistical analysis.

During the observational period 1016 COVID-19 patients were admitted to our Hospital; 194 (19.1\%) met the inclusion criteria. Their clinical characteristics are resumed in Table 1. Of them, 57 patients (29.3\%) developed signs and symptoms of delirium during CPAP. 32 patients $(56.1 \%)$ developed delirium during the first $24 \mathrm{~h}$ after starting CPAP and 48 (84.2\%) in the first $48 \mathrm{~h}$.
Patients developing delirium were older, had a higher frequency of previous diagnosis of dementia or psychiatric condition, a more compromised respiratory function as assessed by $\mathrm{PaO} 2 / \mathrm{FiO} 2$ ratio (immediately after starting CPAP) and a higher neutrophile to lymphocyte ratio (NLR). No significant difference between the two groups were found with respect to comorbidity burden (Charlson Comorbidity Index-CCI), biochemical inflammation-related parameters (IL-6, CRP, D-dimer) nor the use of corticosteroids. The length of hospitalization did not differ, being approximately 20 days in both groups. Time spent in CPAP was highly variable and no statistical significance has been found between the two groups.

Twenty-seven out of 57 patients (47\%) presenting delirium died, accounting for a higher mortality rate in this group. The intubation rate was lower in the delirium group than in patients that didn't develop delirium. Noticeably, delirious patients who underwent ETI were younger than non-intubated delirious ones $(61.4 \pm 13.4$ vs $72.9 \pm 12, \mathrm{p} 0.02)$ and with a trend to a lower comorbidity burden [CCI $<4$ in $5 / 7$ (83\%) of intubated patients vs 19/50 (43\%) in non-intubated patients, p 0.06].

At Cox proportional hazard model, resumed in Table 2, age ( $\mathrm{p}$ 0.04) and $\mathrm{PaO} 2 / \mathrm{FiO} 2$ ratio after starting CPAP ( $\mathrm{p}$ 0.02 ) were significantly correlated with delirium presentation, while NLR and previous diagnosis of dementia or psychiatric conditions didn't reach statistical significance.

Table 1 Clinical and biochemical characteristics of examined population

\begin{tabular}{|c|c|c|c|c|c|}
\hline & General population & $\begin{array}{l}\text { No delirium } 137 \\
70.6 \%\end{array}$ & $\begin{array}{l}\text { Delirium } \\
57 \\
29.4 \%\end{array}$ & $P$ value univariate & Odds ratio univariate \\
\hline Age (years) & $64.0 \pm 14.7$ & $60.9 \pm 14.4$ & $71.5 \pm 12.7$ & $<0.001$ & \\
\hline Age $>70$ years & $77(36.7 \%)$ & $40(29.2 \%)$ & $37(64.9 \%)$ & $<0.0001$ & $4.5(2.3-8.7)$ \\
\hline Male sex & $139(71.6 \%)$ & $100(73.0 \%)$ & $39(68.4 \%)$ & 0.52 & \\
\hline CCI & $2.66 \pm 2.23$ & $2.21 \pm 1.98$ & $3.76 \pm 2.45$ & 0.121 & \\
\hline $\begin{array}{l}\text { Dementia or previous } \\
\text { psychiatric condition }\end{array}$ & $19(9.8 \%)$ & $9(6.6 \%)$ & $10(17.5 \%)$ & 0.024 & $3(1.2-7.9)$ \\
\hline $\mathrm{PaO} 2 / \mathrm{FiO} 2$ at $\mathrm{T} 1$ & $163 \pm 84$ & $173 \pm 84$ & $140 \pm 81$ & $<0.001$ & \\
\hline $\mathrm{PaO} 2 / \mathrm{FiO} 2$ at $\mathrm{T} 1<150$ & $99(51 \%)$ & $60(43.7 \%)$ & $39(68.4 \%)$ & 0.002 & $2.8(1.5-5.3)$ \\
\hline Time in CPAP (hours) & $153.8 \pm 134.4$ & $147.7 \pm 115.8$ & $168.4 \pm 171.4$ & 0.336 & \\
\hline NLR & $9.1 \pm 9.0$ & $7.98 \pm 8.21$ & $11.64 \pm 10.23$ & 0.011 & \\
\hline NLR $>8$ & $55(28.4 \%)$ & $24(17.5 \%)$ & $31(54.9 \%)$ & 0.004 & $2.6(1.4-4.9)$ \\
\hline CRP (mg/L) & $165 \pm 95$ & $157 \pm 90$ & $183 \pm 104$ & 0.088 & \\
\hline D-dimer & $8422 \pm 21,728$ & $6493 \pm 19,545$ & $13,748 \pm 26,396$ & 0.109 & \\
\hline IL-6 & $139 \pm 377$ & $132 \pm 379$ & $161 \pm 377$ & 0.698 & \\
\hline Steroids therapy & $19(9.8 \%)$ & $10(7.3 \%)$ & $9(15.8 \%)$ & 0.07 & \\
\hline Length of stay (days) & $20.6 \pm 14.6$ & $20.8 \pm 15.0$ & $19.1 \pm 13.0$ & 0.52 & \\
\hline Deaths & $59(3.4 \%)$ & $32(23.4 \%)$ & $27(47.4 \%)$ & $<0.001$ & $3(1.5-5.7)$ \\
\hline ETI & $42(21.6 \%)$ & $35(25.5 \%)$ & $7(12.3 \%)$ & 0.041 & \\
\hline
\end{tabular}

In bold the statistically significant data

$T 1$ first data after starting CPAP, CCI Charlson Comorbidity Index, NLR neutrophile to lymphocyte ratio 
Table 2 Cox proportional hazard model: age, previous diagnosis of dementia or psychiatric condition, P/F soon after starting CPAP and NLR

\begin{tabular}{lll}
\hline & $\begin{array}{l}P \text { value multi- } \\
\text { variate }\end{array}$ & Odds ratio multivariate \\
\hline $\begin{array}{l}\text { Age (years) } \\
\begin{array}{l}\text { Dementia or previous } \\
\text { psychiatric condition }\end{array}\end{array}$ & $\mathbf{0 . 0 4}$ & $\mathbf{1 . 0 2 5}(\mathbf{1 . 0 0 8}-\mathbf{1 . 0 4})$ \\
$\mathrm{PaO} 2 / \mathrm{FiO} 2$ at T1 & $\mathbf{0 . 0 2}$ & $1.718(097-2.18)$ \\
$\mathrm{NLR}$ & 0.06 & $\mathbf{0 . 9 9 6}(\mathbf{0 . 9 9 2 - 0 . 9 9 8 )}$ \\
\hline
\end{tabular}

In bold the statistically significant data

The main finding of our work is that almost one third (29.3\%) of a population of consecutive Helmet CPAP ventilated COVID-19 patients presented delirium; this complication was associated with a significantly higher mortality, according to the literature [3-5].

Despite many potential predisposing factors are common in CPAP ventilated patients with AHRF -as hypoxia, dehydration, isolation and noise-delirium incidence in our population seems not to differ from what reported by other studies conducted in populations in which ventilated patients were not represented [3] or constituted a minority of the studied subjects $[4,5]$. Moreover, the occurrence of delirium seems not to be related to prolonged CPAP treatment, as in more than $80 \%$ of the patients it developed in the first $48 \mathrm{~h}$ and no correlation between time spent in CPAP and delirium onset was found.

Our data confirm that age and previous diagnosis of dementia or psychiatric condition can be considered risk factors for the development of delirium [3, 4]. Furthermore, severe respiratory impairment is associated to delirium in our cohort as $\mathrm{PaO} 2 / \mathrm{FiO} 2$ ratio was statistically lower in delirious patients. This latter finding is in accordance with previous work reporting an increased risk for neuropsychiatric symptoms in patients with more severe COVID-19 disease and worst respiratory failure [1].

A significantly lower intubation rate was observed in patients who developed delirium; anyway, delirious patients were older with higher prevalence of dementia, conditions that limit ETI's indication. For this reason and for possible selection bias we can't consider this result as accurate.

The study presents some limitations. First, it is possible that patients at greater risk for delirium (i.e. elderly with severe dementia) were not ventilated, thus making possible an under-estimation of delirium occurrence when compared to non-ventilated patients. Moreover, populations in comparison found in literature were not completely comparable to ours for age, severity of illness and settings. For these reasons this data should be taken carefully. For last, our study was performed in a single center dedicated to high-complexity medical care and our results should not be generalized to different populations.
In conclusion CPAP therapy may not increase delirium occurrence, as indicated by an incidence in our cohort of non-invasive ventilated patients similar to what found in other works regarding non-ventilated COVID-19 subjects, even if further studies should be done to confirm this data. Unfortunately, COVID-19 pandemic, especially during the first wave, drastically challenged hospital organization, forcing to the use of Helmet CPAP also outside ICU or sub-intensive units, where monitoring patients is more difficult. Our study may add evidences for the recognition of patients at high risk for delirium (over 70 years; mentally ill; NLR ratio $>8$; $\mathrm{P} / \mathrm{F}<150 \mathrm{mmHg}$ after starting $\mathrm{CPAP}$ ) needing a strict supervision and the highest intensity of care setting for at least the first $48 \mathrm{~h}$.

\section{Declarations}

Conflict of Interest No conflict of interest is present as already declared in the appropriate model.

Human and animal rights statement The study is part of a prospectively conducted registry study ("REGISTRO DELLE INFEZIONI SOSPETTE E ACCERTATE COVID-19/Studio Sacco COVID-19") which was approved by the Local Ethical Committee with the Registration Number 2020/16088.

Informed consent All participants provided informed consent prior to their participation.

\section{References}

1. Mao L, Jin H, Wang M et al (2020) Neurologic manifestations of hospitalized patients with Coronavirus Disease 2019 in Wuhan, China. JAMA Neurol 77(6):683-690. https://doi.org/10.1001/jaman eurol.2020.1127

2. Rogers JP, Chesney E, Oliver D et al (2020) Psychiatric and neuropsychiatric presentations associated with severe coronavirus infections: a systematic review and meta-analysis with comparison to the COVID-19 pandemic. Lancet Psychiatr 7(7):611-627. https://doi. org/10.1016/S2215-0366(20)30203-0

3. Poloni TE, Carlos AF, Cairati M et al (2020) Prevalence and prognostic value of Delirium as the initial presentation of COVID-19 in the elderly with dementia: an Italian retrospective study. EClinicalMedicine 26:100490. https://doi.org/10.1016/j.eclinm.2020.100490

4. Garcez FB, Aliberti MJR, Poco PCE et al (2020) Delirium and adverse outcomes in hospitalized patients with COVID-19. J Am Geriatr Soc. https://doi.org/10.1111/jgs.16803

5. Marengoni A, Zucchelli A, Grande G, Fratiglioni L, Rizzuto D (2020) The impact of delirium on outcomes for older adults hospitalised with COVID-19. Age Ageing. https://doi.org/10.1093/ageing/afaa189/5894896 (Oxford Univ Press)

Publisher's Note Springer Nature remains neutral with regard to jurisdictional claims in published maps and institutional affiliations. 